\title{
DEVELOPMENT OF VERSATILE MOBILE MAPPING SYSTEM ON A SMALL SCALE
}

\author{
T. Tachi ${ }^{1 *}$, Y. Wang ${ }^{1}$ R. Abe ${ }^{1}$, T. Kato ${ }^{1}$, N. Maebashi ${ }^{1}$, N. Kishimoto ${ }^{1}$ \\ ${ }^{1}$ Geospatial Technology Department, Kokusai Kogyo Co., Ltd., 2-24-1 Harumi-cho, Fuchu, Tokyo 183-0057, Japan - \\ (tomoe_tachi, a_o, ryogo_abe, takayuki_kato, naoya_maebashi, natsuko_kishimoto) @ kk-grp.jp
}

Commission I, WG I/7

KEY WORDS: Mobile mapping system, Pegasus II, field-portable, accuracy evaluation, terrain survey, facility inspection

\begin{abstract}
:
Mobile mapping technology is an effective method to collect geospatial data with high point density and accuracy. It is mainly used for asset inventory and map generation, as well as road maintenance (detecting road cracks and ruts, and measuring flatness). Equipment of former mobile mapping systems (MMS) is large in size and usually installed (hard-mounted) onto dedicated vehicle. Cost-effectiveness and flexibility of MMS have not been regarded as important until Leica Pegasus series, a much smaller system with integrated and configurable components, come out. In this paper, we show you how we realize a versatile MMS with a Pegasus II loaded on a remodelled Japanese light vehicle (small size and less than a cubic capacity of $660 \mathrm{cc}$ ). Besides Pegasus II and dataprocessing PC, we equip this system with a small crane to bring the sensor onto a different platform, an electric cart to survey narrow roads or pedestrian walkway, and a boat attachment so that the sensor can be fixed on a boat. Thus, one Pegasus II can collect data from various platforms. This paper also discusses the precision and accuracy of the Pegasus II working on various platforms. When mounted on a light vehicle, we verified the accuracy of the difference with GCP and evaluated the accuracy of the road maintenance (detecting road cracks and ruts, and measuring flatness). When mounted on an electric cart, we verified the accuracy of the difference with GCP on a pedestrian road and generated road hazard map as a data utilization. When mounted on a boat, we verified the accuracy of the difference with GCP on a dam slope and created slope shading map of landslide area as a data utilization. It turns out that Pegasus II can totally achieve to required surveying-grade.
\end{abstract}

\section{INTRODUCTION}

Mobile mapping technology is an effective method to collect geospatial data with high point density and accuracy. It is mainly used for asset inventory and map generation, as well as road maintenance such as detecting road cracks and ruts, and measuring flatness. Equipment of former mobile mapping systems (MMS) is large in size and usually hard-mounted onto dedicated vehicle.

Recently developed portable equipment for MMS are much smaller and lighter than conventional large-sized models, and can be flexibly mounted on various platforms such as vehicles, trolleys, and boats according to various purposes. However, since a highly accurate portable equipment of MMS is relatively heavy and has a relatively large structure, it requires a substantial amount of efforts for transportation and installation during field work as well. Cost-effectiveness and flexibility of MMS have not been regarded as important until versatile portable multi-sensor equipment Leica Geosystems Pegasus series, much smaller equipment with integrated and configurable components, come out.

Accordingly, we decided to develop a versatile accurate MMS on a small scale with a Leica Geosystems Pegasus II as measuring equipment. Our developed system adopted a light automobile as a transportation vehicle and one of platforms. A trolley and a boat can be utilized as a platform of the developed system according to various purposes. In this paper, we show you a brief outline, measurement accuracy, and various application of the developed system.

\section{OUTLINE OF THE DEVELOPED SYSTEM}

We aimed that to develop a versatile accurate MMS that should be smaller, lighter, more cost-effective, and more feasible.

\subsection{Measuring equipment}

We adopted a Leica Geosystems Pegasus II shown in Figure 1 as measuring equipment of our developed system. The Pegasus II is portable multi-sensor equipment integrating measuring devices with position devices. Table 1 shows the specifications of the Pegasus II. the Pegasus II has 8 cameras and a laser scanner as measuring devices and one ow two GNSS signal receivers, an inertial measurement unit (IMU) and a distance measurement instrument (DMI) as positioning devices. It is approved to be utilized for the Japanese public surveying of the map information level 500 of which the accuracy requirement is that the maximum measurement error in horizontal and vertical directions should be equal or less than $15 \mathrm{~cm}$ and $20 \mathrm{~cm}$ respectively.

Furthermore, since all measuring and position devices are integrated, individual calibration of each device is unnecessary. On the other hand, although the Pegasus II is lighter than former highly accurate integrated measuring equipment, the weight is still $51 \mathrm{~kg}$ for the measuring and positioning devices and in addition $30 \mathrm{~kg}$ for the battery. Therefore, when carrying

\footnotetext{
* Corresponding author
} 
out on-site work on a trolley or a boat, besides a transportation vehicle, two or more persons for installing the equipment on a platform are required. In case of measurement from a roof of a vehicle, installation and removal of the equipment takes not a little time.

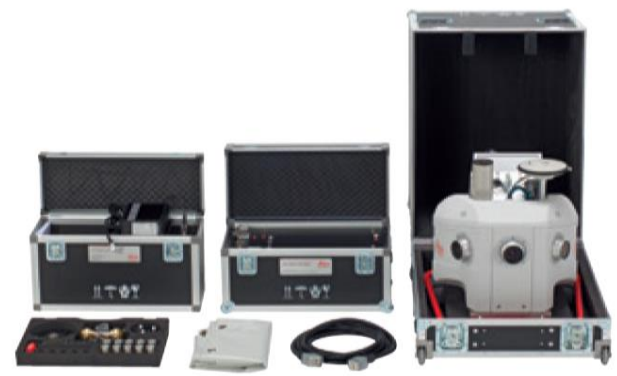

Figure 1. Leica Geosystems Pegasus II

\begin{tabular}{|l|l|}
\hline Camera & $\begin{array}{l}\text { 8 (1 rear down facing camera) } \\
\text { 2000 pixels x 2000 pixels }\end{array}$ \\
\hline Laser scanner & ZF9012 profiler \\
& (phase-based laser scanner) \\
& Scan rate:1,016Hz \\
& Scan speed:200Hz \\
& Linearity error:1mm \\
\hline Positioning device & GNSS: Max. 2 \\
& IMU: 1 \\
& DMI: 1 \\
\hline Position accuracy after 10 & 0.020 m RMS horizontal \\
sec. of outage duration & 0.020 m RMS vertical \\
& 0.008 degrees RMS pitch/roll \\
& 0.013 degrees RMS heading \\
\hline Weight & $51 \mathrm{~kg}$ \\
\hline Size & $60 \times 79 \times 76 \mathrm{~cm}$, \\
\hline
\end{tabular}

Table 1. Specifications of Leica Geosystems Pegasus II

\subsection{Hardware configuration of the developed system}

The Pegasus II does not require a designated vehicle, and is small and light enough to transport and mount on various small platforms such as a small vehicle, a trolley, a boat. However, at present, a dedicated frame to mount the Pegasus II is required when a small vehicle, a trolley, or a boat is utilized as a platform. Moreover, a van-type vehicle for transporting the Pegasus II is necessary when using a trolley, or a boat as a platform. Although the Pegasus II is small and light, it is not easy for one or two persons to mount it on a platform on-site.

Therefore, we designed a MMS on a small scale that integrates measuring equipment, a transportation vehicle, and various platforms such as a light vehicle, a trolley, and a boat. Table 2 shows the hardware configuration of our designed system.

\begin{tabular}{|l|l|}
\hline $\begin{array}{l}\text { Measuring } \\
\text { equipment }\end{array}$ & Leica Geosystems Pegasus II \\
\hline $\begin{array}{l}\text { Transportation } \\
\text { vehicle }\end{array}$ & $\begin{array}{l}\text { Remodeled Daihatsu Hijet } \\
\text { (Japanese mini deck van) } \\
\text { with a small crane to bring the Pegasus II } \\
\text { onto various platforms }\end{array}$ \\
\hline $\begin{array}{l}\text { Observation } \\
\text { platform }\end{array}$ & $\begin{array}{l}\text { light vehicle } \\
\text { (the same as the transportation vehicle) } \\
\text { trolley with assist of electric motor } \\
\text { boat }\end{array}$ \\
\hline
\end{tabular}

Table 2. Hardware configuration of the developed system
We adopted a remodelled Japanese light vehicle, of which size is $3.4 \mathrm{~m} \mathrm{x} 1.48 \mathrm{~m} \times 1.9 \mathrm{~m}$, and displacement is $658 \mathrm{cc}$, as a transportation vehicle and a platform. The vehicle has a small crane installed at the rear of the loading board to reduce labour for transportation, mounting, and measurement. As for a trolley as a platform, an electric motor was attached in order to moving easily against the a little heavy weight of the Pegasus II with a battery. Additionally we standardized a mounting frame for various platforms. The standardized frame has a rotating table and a lifter for easy operation and safely transporting the Pegasus II.

Figure 2 shows how to mount the Pegasus II on various platforms. Figure 3 shows our developed system to achieve versatile mobile mapping.

From now on, the system using a vehicle as a platform is called the vehicle-loading system. Similarly, the trolley-loading system and the boat-loading system mean the system using a trolley as a platform and the system using a boat as a platform, respectively.

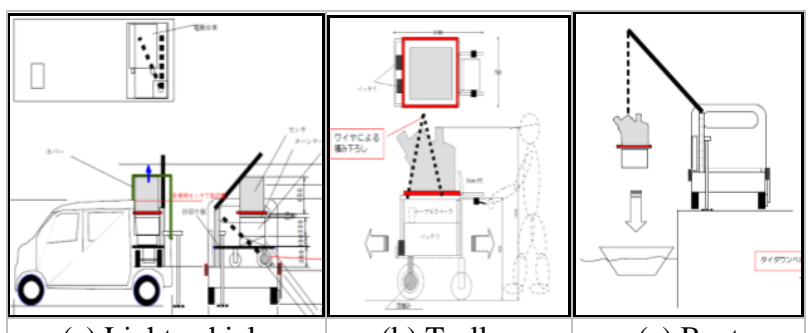

(a) Light vehicle

(b) Trolley

(c) Boat

Figure 2. Mounting Pegasus II on various platforms

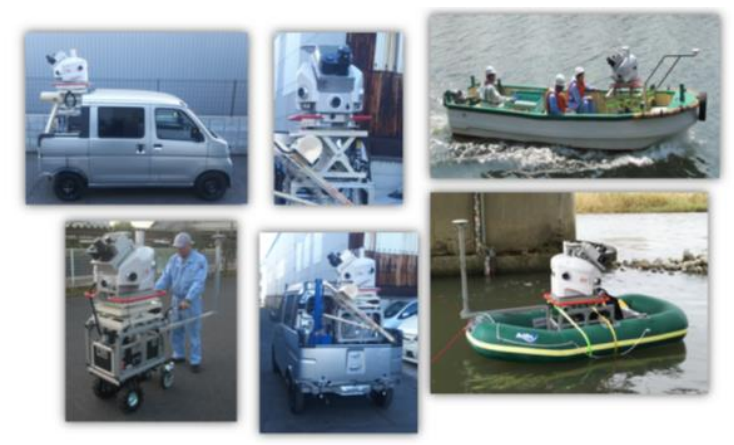

Figure 3. Developed system to achieve versatile mobile mapping

\section{EVALUATION OF THE DEVELOPED SYSTEM}

We evaluated the measurement accuracy by the developed system in using various platforms.

\subsection{Developed vehicle-loading system}

The evaluation on the accuracy of the developed vehicleloading system was conducted at a suburb of Tokyo as Figure 4 shows. We selected 225 feature points as check points of the evaluation. A feature adjacent to a road was selected as a check point. A typical check point was a corner of a road marking as Figure 5 shows. 


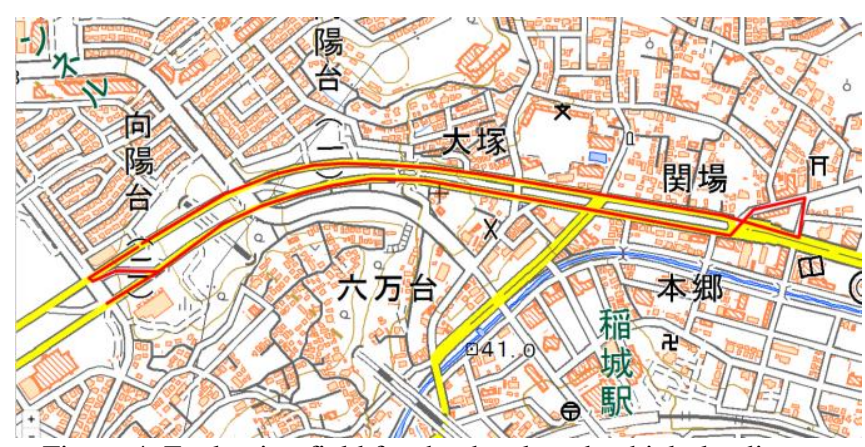

Figure 4. Evaluation field for the developed vehicle-loading system
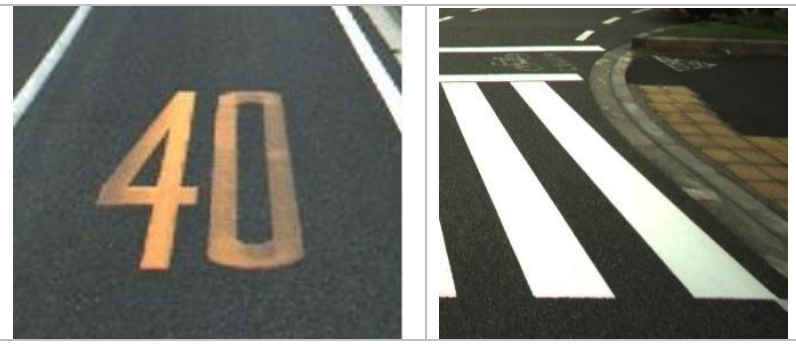

Figure 5. Typical check points for evaluation of the developed vehicle-loading system

Figure 6 shows a reflection intensity image of LiDAR data utilized for evaluation of the developed system. The evaluation on the measurement accuracy was carried out by comparing check points' positions observed by the vehicle-loading system with those measured by network real time kinematic (RTK) GNSS surveying.

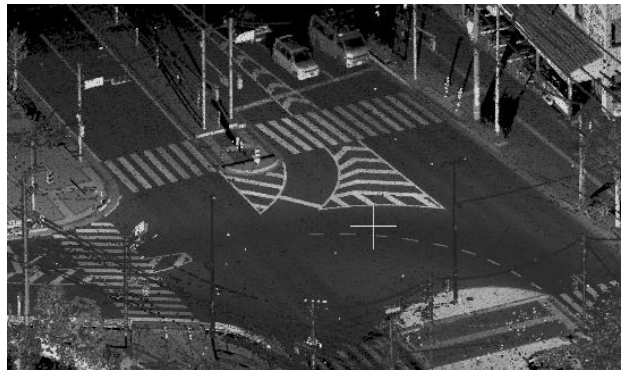

Figure 6. LiDAR data for evaluation of the developed vehicleloading system

Table 3 shows results of the accuracy evaluation of the vehicleloading system. The root mean squares of errors (RMSE) in horizontal direction was $6.8 \mathrm{~cm}$, while that in vertical direction was $7.9 \mathrm{~cm}$. The results shows that the developed vehicleloading system would be able to satisfy the accuracy requirement of the map information level 500 of the Japanese public surveying that the maximum difference in horizontal and vertical direction should be equal or less than $15 \mathrm{~cm}$ and $20 \mathrm{~cm}$ respectively.

\begin{tabular}{|l|c|c|}
\hline & Horizontal $(\mathrm{m})$ & Vertical $(\mathrm{m})$ \\
\hline Maximum & 0.125 & 0.134 \\
\hline Average & 0.062 & 0.074 \\
\hline RMSE & 0.068 & 0.079 \\
\hline
\end{tabular}

Table 3. Results of accuracy evaluation of the developed vehicle-loading system

\subsection{Developed trolley-loading system}

The evaluation on the accuracy of the developed trolley-loading system was conducted at a suburb of Oosaka. We selected 24 feature points as check points of the evaluation. A feature adjacent to a road was selected as a check point in the same as the evaluation of the vehicle-loading system. A typical check point was a corner of a white line on a road as Figure 7 shows.
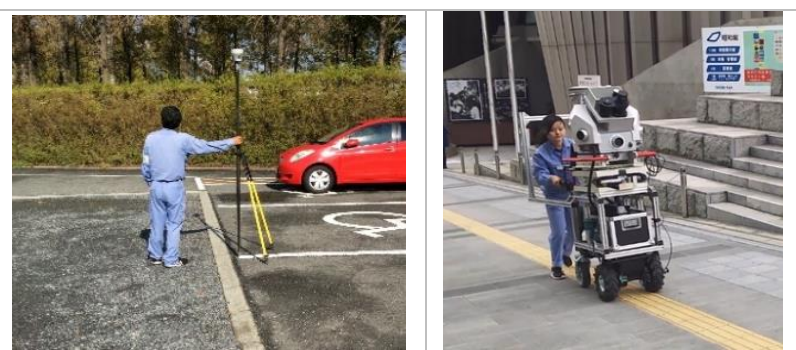

Figure 7. Typical check points (left) for evaluation of the developed trolley-loading system (right)

Figure 8 shows a reflection intensity image of LiDAR data utilized for evaluation of the trolley-loading system. The evaluation on the measurement accuracy was carried out by comparing check points' positions observed by the vehicleloading system with those measured by network RTK GNSS surveying.

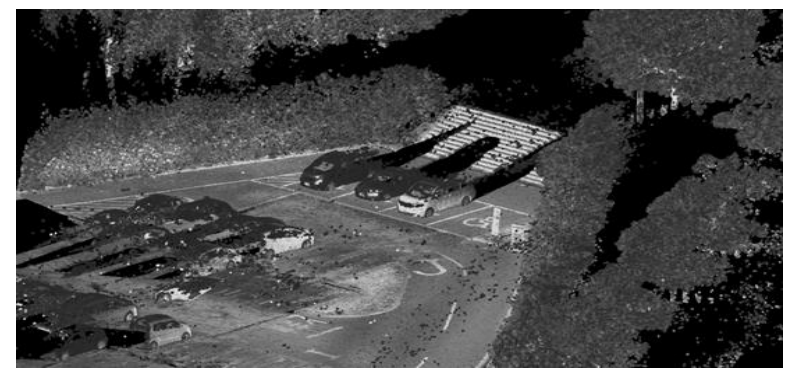

Figure 8. LiDAR data for evaluation of the developed trolleyloading system

Table 4 shows results of the accuracy evaluation of the trolleyloading system. The RMSE in horizontal direction was $5.5 \mathrm{~cm}$, while that in vertical direction was $1.7 \mathrm{~cm}$. The results shows that the developed trolley-loading system would be able to satisfy the accuracy requirement of the map information level 500 of the Japanese public surveying.

\begin{tabular}{|l|c|c|}
\hline & Horizontal $(\mathrm{m})$ & Vertical $(\mathrm{m})$ \\
\hline Maximum & 0.065 & 0.033 \\
\hline Average & 0.054 & 0.018 \\
\hline RMSE & 0.055 & 0.017 \\
\hline
\end{tabular}

Table 4. Results of accuracy evaluation of the developed vehicle-loading system

However, distortion of LiDAR data observed by the trolleyloading system was found around a place where the attitude of the sensor changes abruptly such as a sharp curve. Observation data by the vehicle-loading system had no such distortion. The cause of the distortion might be slower moving speed around a sharp curve.

Moreover, when conducting measurement for a long distance for instance longer than $2 \mathrm{~km}$, the amount of observed data becomes significantly large, and processing work afterwards 
takes too much time. Assuming actual operation, we would like to suggest that measurement should be conducted along a straight line as much as possible, with an optimum distance at approximately $500 \mathrm{~m}$, and the maximum distance at approximately $2 \mathrm{~km}$.

\subsection{Developed boat-loading system}

The evaluation on the accuracy of the developed boat-loading system was conducted at a lake in a suburb of Tokyo.

We set up 18 check points on a slope of a dam for the evaluation. A target of each check point was $50 \mathrm{~cm} \times 50 \mathrm{~cm}$ square white panel, and 18 check points were arranged 3 columns by 6 rows as Figure 9 shows. A centre of a panel was treated as a positon of each check point, and its positon was measured by network RTK GNSS surveying.

On the other hand, we extracted points belonging in each check point from LiDAR point cloud obtained by the developed system. The centre of gravity of extracted point for each check point was treated as a measured positon of the check point.
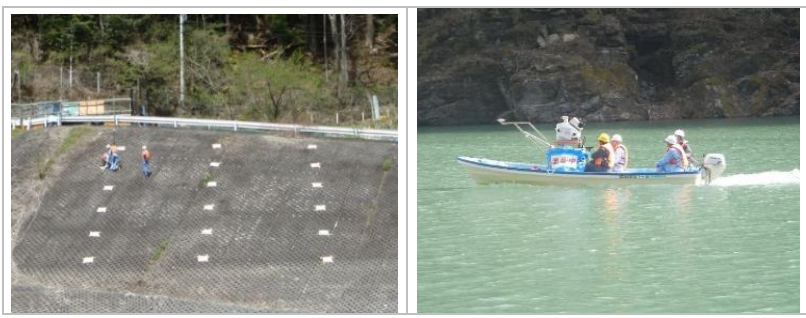

Figure 9. Accuracy evaluation of the developed boat-loading system

Table 5 shows results of the accuracy evaluation of the boatloading system. The RMSE in horizontal direction was $7.0 \mathrm{~cm}$, while that in vertical direction was $3.6 \mathrm{~cm}$. The results shows that the developed boat-loading system would be able to satisfy the accuracy requirement of the map information level 1000 of the Japanese public surveying that the maximum difference in horizontal and vertical direction should be equal or less than $30 \mathrm{~cm}$ and $30 \mathrm{~cm}$ respectively.

\begin{tabular}{|l|c|c|}
\hline & Horizontal (m) & Vertical (m) \\
\hline Maximum & 0.288 & 0.140 \\
\hline Average & 0.165 & 0.080 \\
\hline RMSE & 0.070 & 0.036 \\
\hline
\end{tabular}

Table 5. Results of accuracy evaluation of the developed boatloading system

However, although the measurement accuracy of the boatloading system in total was high enough, the measurement accuracy in the upper part of the slope was rather low. The reason of the accuracy degradation in the upper part of the slope would be that the incident angle became shallower toward the upper part of the slope and the point density became lower as Figure 10.

Moreover, when a place was measured several times in a deep valley, there found a few differences of more than $1 \mathrm{~m}$ between measurement results. It is considered that this is because the GNSS signal receiving status was poor and the correction by the DMI was unable to be utilized. Accordingly, the measurement of the position and attitude of the sensor was performed only by the IMU. We considered that since the running speed of the boat was slow, the measurement error of the IMU would be accumulated, and the measurement accuracy of the position and attitude of the sensor would have decreased.

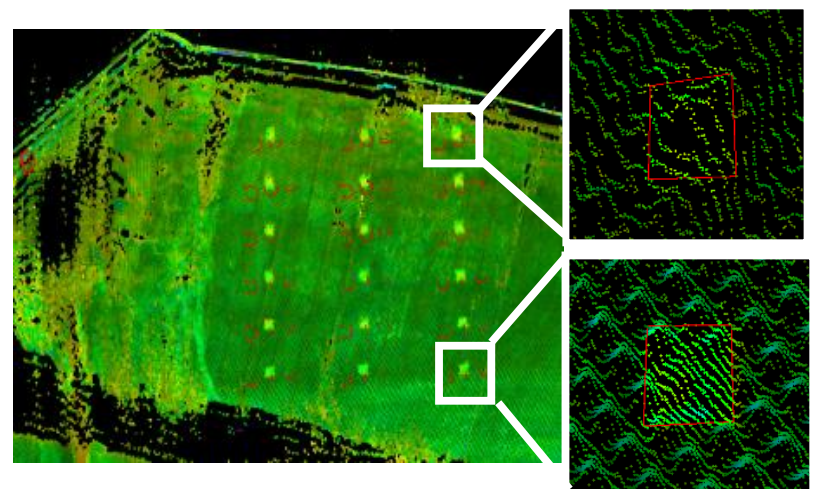

Figure 10. LiDAR data for evaluation of the developed boatloading system

\section{APPLICATION OF THE DEVELOPED SYSTEM}

We show you various application of our developed system.

\subsection{Developed vehicle-loading system}

Most of infrastructures in Japan such as roads, railways, ports, channels, and power lines, were designed and built in the highgrowth period between the late 60's and mid 70's. These infrastructures are now aging and deteriorated. Therefore, periodical inspection of the infrastructures is extremely required. A significant cost such as time and labour force involved in inspection is data collection. However decreasing and aging population in Japan leads to a shortage of labour forces, and severe fiscal constraint leads to a declining expenditure on public utilities. Means to collect precise data quickly, safely and less costly is required.

Our developed system would be a promising means for quick, safe, and efficient inspection. Figure 11 shows an application of the developed vehicle-loading system utilized in road surface inspection. The developed system can measures the following items indicating a road surface state:

(1) Cracks: Images obtained by the rear down facing camera of the Pegasus II are utilized for detection of cracks on a road surface. Sunlight is utilized as a light source for imaging in the daytime.

(2) Ruts: The laser scanner of the Pegasus II provides a point cloud to measure the depth of ruts.

(3) Unevenness of longitudinal profile: Longitudinal profile is measured by using observation data of the positions devices such as GNSS, IMU, and DMI of Pegasus II.

Odometry in the developed system is carried out by the DMI attached to a rear wheel of a vehicle.

The vehicle-loading system acquired a Japanese official certification of road surface inspection approved by the Public Works Research Center in Japan. The certification shown in Figure 12 indicates that the vehicle-loading system sufficiently satisfies the requirements for road surface inspection conducted in the daytime in Japan. 


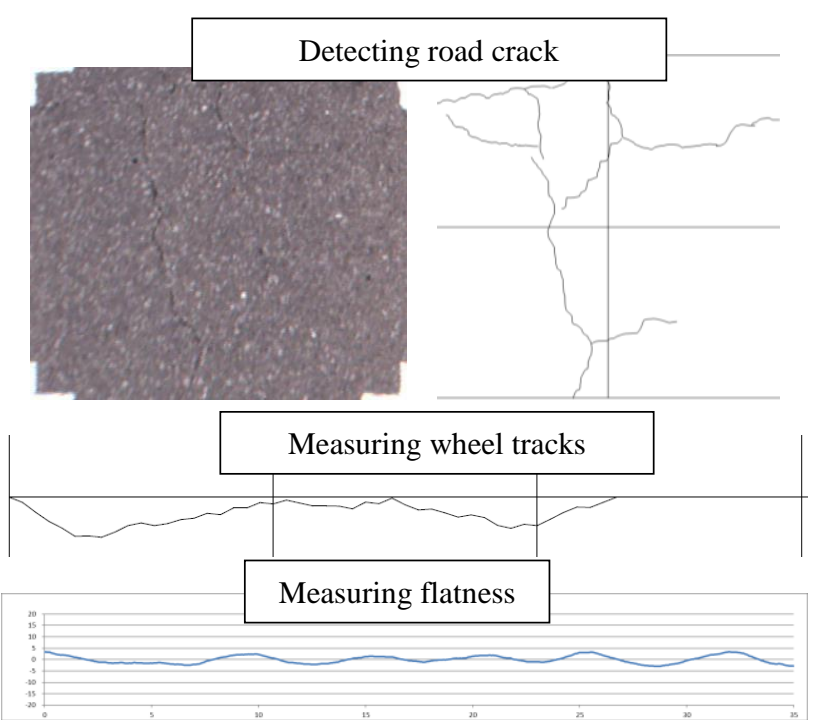

Figure 11. Application of the developed vehicle-loading system utilized in road surface inspection
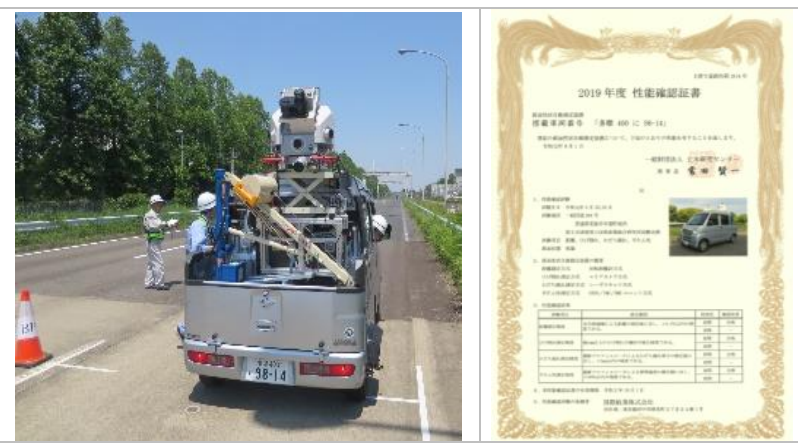

Figure 12. Developed vehicle-loading system for road surface inspection and Japanese official certification of road surface inspection

\subsection{Developed trolley-loading system}

According to the Ministry of Health, Labour and Welfare of Japan (MHLW), 12.2\% of Japanese over 65 died from injuries by falling. This number would increase along with ageing population problem. Accordingly generation of road hazard maps of pedestrian walkways has been required.

Since the developed trolley-loading system can collect road data where ordinary vehicle cannot enter, like pedestrian walkways, it would be effective for generating road hazard maps for pedestrians. A road hazard map indicates where in a walkway is danger to a pedestrian. The trolley-loading system can provide LiDAR data to detect places such as uneven parts that are difficult for pedestrians to walk. Figure 13 shows an application of the trolley-loading system utilized in generation of a road hazard map.

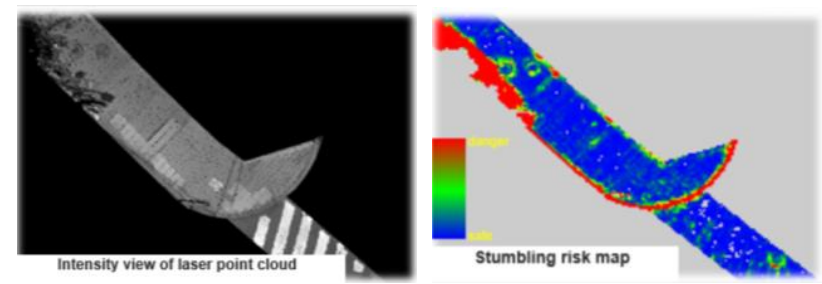

Figure 13. Application of the developed trolley-loading system utilized in generation of a road hazard map

\subsection{Developed boat-loading system}

Regular inspection of terrain around a dam is important to early detection and prevention of sediment and geological disasters, as well as flood control. In Japan, the Ministry of Land, Infrastructure, Transport and Tourism of Japan (MLIT) and dam management offices conduct terrain surveying by airborne laser scanning (ALS) every 5 years for dams. However, as for terrain change on a small scale, like a small landslide or erosion caused by rain, ALS is not cost-effective. ALS is also limited to surveying a slope of which terrain is depressed in vertical direction because it scans terrain from upside.

On the other hand, our developed boat-loading system can be utilized in terrain surveying for dam management. The boatloading system can scan a dam slope from downside, thus, it can be a supplement of ALS to capture 3D terrain of the dam. Figure 14 shows an application of the boat-loading system utilized to terrain surveying a dam.
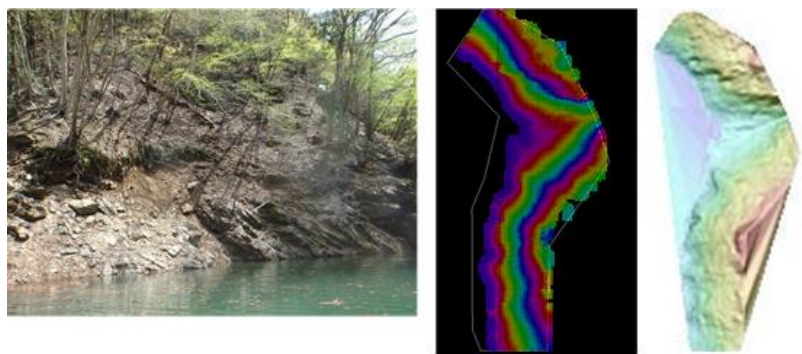

Figure 14. Application of the developed boat-loading system utilized in terrain surveying around a dam

\section{SUMMARY}

We developed a versatile accurate MMS on a small scale with a Leica Geosystems Pegasus II as measuring equipment. Our developed system utilizes a remodelled Japanese light automobile as a transportation vehicle and one of platforms. We equipped the transportation vehicle with a small crane to bring the sensor onto various platforms such as an electric trolley to survey narrow roads or pedestrian walkway, and a boat to survey from the surface of the water.

The measurement accuracy by the vehicle-loading system and the trolley-loading system would be able to satisfy the accuracy requirement of the map information level 500 of the Japanese public surveying. On the other hand, although the measurement accuracy by the boat-loading system would be a little bit lower and be able to satisfy the accuracy requirement of the map information level 1000 of the Japanese public surveying.

We consider that the developed system would be one of MMSs which are accurate, small, light, cost-effective, and feasible for various application. 